\title{
Exploring the Interaction between E484K and N501Y Substitutions of SARS-CoV-2 in Shaping the Transmission Advantage of COVID-19 in Brazil: A Modeling Study
}

\author{
Shi Zhao, ${ }^{1,2^{*}}$ Jinjun Ran, ${ }^{3}$ and Lefei $\operatorname{Han}^{4 *}$ \\ ${ }^{1}$ JC School of Public Health and Primary Care, Chinese University of Hong Kong, Hong Kong, China; ${ }^{2}$ CUHK Shenzhen Research Institute, \\ Shenzhen, China; ${ }^{3}$ School of Public Health, Shanghai Jiao Tong University School of Medicine, Shanghai, China; ${ }^{4}$ School of Global Health, \\ Chinese Center for Tropical Diseases Research, Shanghai Jiao Tong University School of Medicine, Shanghai, China
}

\begin{abstract}
The COVID-19 pandemic poses serious threats to global health, and the emerging mutation in SARSCoV-2 genomes is one of the major challenges of disease control. Considering the growth of epidemic curve and the circulating SARS-CoV-2 variants in Brazil, the role of locally prevalent E484K and N501Y substitutions in contributing to the epidemiological outcomes is of public health interest for investigation. We developed a likelihood-based statistical framework to reconstruct reproduction numbers, estimate transmission advantage associated with different SARS-CoV-2 variants regarding the marking (identifying) $484 \mathrm{~K}$ and $501 \mathrm{Y}$ substitutions (including Alpha, Zeta, and Gamma variants) in Brazil, and explored the interactive effects of genetic activities on transmission advantage marked by these two mutations. We found a significant transmission advantage associated with the 484K/501Y variants (including P.1 or Gamma variants), which increased the infectivity significantly by $23 \%$. In contrast and by comparison to Gamma variants, E484K or N501Y (including Alpha or Zeta variants) substitution alone appeared less likely to secure a concrete transmission advantage in Brazil. Our finding indicates that the combined impact of genetic activities on transmission advantage marked by $484 \mathrm{~K} / 501 \mathrm{Y}$ outperforms their independent contributions in Brazil, which implies an interactive effect in shaping the increase in the infectivity of COVID-19. Future studies are needed to investigate the mechanisms of how E484K and N501Y mutations and the complex genetic mutation activities marked by them in SARS-CoV-2 affect the transmissibility of COVID-19.
\end{abstract}

\section{INTRODUCTION}

COVID-19, the etiological agent of which is severe acute respiratory syndrome coronavirus 2 (SARS-CoV-2), ${ }^{1}$ posed a serious threat to global health and swept the world in 2020; the pandemic is still ongoing. ${ }^{2,3}$ As of March 31, 2021, more than 127 million COVID-19 cases had been confirmed worldwide, with more than 2.7 million associated deaths.

The control of COVID-19 requires knowledge of the driving factors that may affect the transmission process $^{4,5}$; virus mutation is one of the major challenges. ${ }^{6,7}$ Around September 2020, genetic variants carrying the N501Y substitution on the spike (S) protein of SARS-CoV-2 were first detected in the United Kingdom ${ }^{8}$ and, then spread globally and trended to reach fixation rapidly in many places (e.g., South Africa, ${ }^{9}$ Brazil, ${ }^{10}$ the United States, ${ }^{11}$ and the United Kingdom). ${ }^{12,13}$ In Brazil, the $501 Y$ variants, as well as other amino acid changes, were clustered into the B.1.1.28.1 lineage by COVID-19 Genomics Consortium UK, which is also known as the variant of concern (VoC) 202101/02. ${ }^{14}$ The B.1.1.28.1 lineage is a descendant of the B.1.1.28 lineage that has another similar descendant lineage, B.1.1.28.2, carries the E484K substitution but not N501Y substitution. ${ }^{15,16}$ The mutation E484K was first identified in South Africa and became prevalent in many places, including the United Kingdome and Brazil. ${ }^{17}$ These emerging variants may affect the epidemiological characteristics of COVID-1918,19 and the protective effects of vaccines. ${ }^{20-23}$ Considering the growing patterns of the epidemic curve in Brazil, the possible contributions of both E484K and N501Y substitutions are of public health interest for investigation.

*Address correspondence to Shi Zhao, Rm.: 502, 5/F, Public Health Building, Prince of Wales Hospital, ST, NT, Hong Kong, Email: zhaoshi.cmsa@gmail.com or Lefei Han, Rm.: 407, Building No. 1, 280 South Chongqing Rd., Shanghai, China, E-mail: Ifhan@ sjtu.edu.cn.
The rapid spread of $501 \mathrm{Y}$ and $484 \mathrm{~K}$ variants indicates a possible transmission advantage over their preceding variants. ${ }^{24}$ On one hand, recent analyses reported evidence that the N501Y substitution was associated with an increase in infectivity of COVID-19, ${ }^{12,13,25-27}$ which appears similar to the situation of D614G substitution reported previously. ${ }^{28-31}$ On the other hand, the relationship between E484K substitution and COVID-19 transmissibility appears inconclusive. ${ }^{32,33}$ The survival or functional profile of pathogen could be altered through genetic mutation and, as a consequence, change its infectivity. ${ }^{34}$ Referring to the previous studies on seasonal influenza viruses, ${ }^{35}$ a few key amino acid substitutions may lead to changes in antigenic features and epidemiological outcomes, ${ }^{36,37}$ and the interaction among them may become more complicated. As an example, the R384G substitution in the nucleoprotein (NP) of H3N2 virus enhances the ability of in-host immune escape, ${ }^{38}$ which increases transmissibility, ${ }^{35}$ but this substitution appears detrimental. In contrast, the commutations including E375G and M239V in NP could compensate and restore the viral fitness or functionality of H3N2 virus, ${ }^{39,40}$ just as the mutated strains rapidly reached fixation in 1993-1994 influenza season. For the COVID-19 epidemics in Brazil, how the mutation activities marked by E484K and N501Y substitutions, as well as the possible interactive effect between them, might shape the transmission advantage remains unassessed.

Exploring the role of mutation activities in determining disease transmissibility is of importance to understand how the evolutionary process at molecular scale may shape the epidemiological outcomes at population scale. ${ }^{28,31,41,42}$ In this study, we adopt a statistical framework to infer the real-time transmissibility associated with different SARS-CoV-2 variants with respect to E484K and N501Y substitutions in Brazil. We explore the interactive effects between E484K and N501Y in shaping the transmission advantage of COVID-19. 


\section{METHODS}

SARS-CoV-2 sequencing data and COVID-19 surveillance data. The SARS-CoV-2 strains were obtained via the global initiative on sharing all influenza data (GISAID) with collection dates ranging from January 1, 2020 to January 31, 2021 , in the Brazil. ${ }^{43}$ A total of 4,210 complete human SARS-CoV-2 strains were retrieved. We excluded sequences with more than $5 \%$ ambiguous amino acids during the alignment, and a total of 4,052 sequences were included for further analysis. Multiple sequence alignment was performed using MAFFT version $7,{ }^{44}$ and the "Wuhan-Hu-1" (GISAID: EPI_ISL_402125 or GenBank: NC_045512.2) SARS-CoV-2 genome is considered as the reference sequence.

The surveillance data of COVID-19 cases in the Brazil were collected via the WHO COVID-19 surveillance platform. ${ }^{45}$ To avoid the under-ascertainment due to reporting delay, we drop the observations since February 2021. As such, the surveillance data of COVID-19 cases from January 1, 2020 to January 31,2021 , are included in the analysis, which match the period of SARS-CoV-2 sequencing data. To adjust for the weekly cycle in the COVID-19 case time series, the 7-day moving average is adopted for further analysis. The COVID19 cases time series are shown in Figure 1A.

Statistical parameterization. Variant-specific reproduction number. The time-varying reproduction number is commonly adopted to quantify the instantaneous transmissibility of infectious disease in an epidemic. Using the estimation framework in Cori et al., ${ }^{46}$ the epidemic growth is modeled as a branching process. Thus, the reproduction number at time $t, R(t)$, is expressed as the ratio of $C(t)$ over $\int_{0}^{\infty} w(\tau) C(t-\tau) \mathrm{d} \tau$, which is commonly known as the renewable equation. ${ }^{47,48}$ Here, the $C(t)$ is the observed new incidences of COVID-19 at time $t$. The function $w(\cdot)$ is the distribution of the generation time (GT) of the disease, that is, COVID-19. The GT is defined as the time interval between the time of exposure, that is, being infected, of a primary case and that of his associated secondary case in the consecutive transmission generation. ${ }^{49}$ Thus, the distribution $w(\cdot)$ is predefined in our model, which is commonly estimated from contract tracing surveillance data. ${ }^{50-53}$ To set up the analysis for COVID-19, we consider $w$ as a Gamma distribution having mean $( \pm S D)$ values of $5.3( \pm 2.1)$ days by averaging the GT estimates from the existing literatures. ${ }^{50-57}$ Slight variation in the settings of the GT will not affect our main findings.

To incorporate the information of SARS-CoV-2 variants, we denote the proportion (or prevalence) of the $j$-th variant of concern (VoC) at calendar time $t$ by $\rho_{j}(t)$, which is timevarying. Straightforwardly, $\sum_{j} \rho_{j}(t)=1$ for all $t$. We denote the variant-specific reproduction number for the $j$-th $\mathrm{VoC}$ at time $t$ by $R_{j}(t)$, and we have $R_{j}(t)=\frac{\rho_{j}(t) C(t)}{\int_{0}^{\infty} w(\tau) \rho_{j}(t-\tau) C(t-\tau) \mathrm{d} \tau}$. We

\section{C}
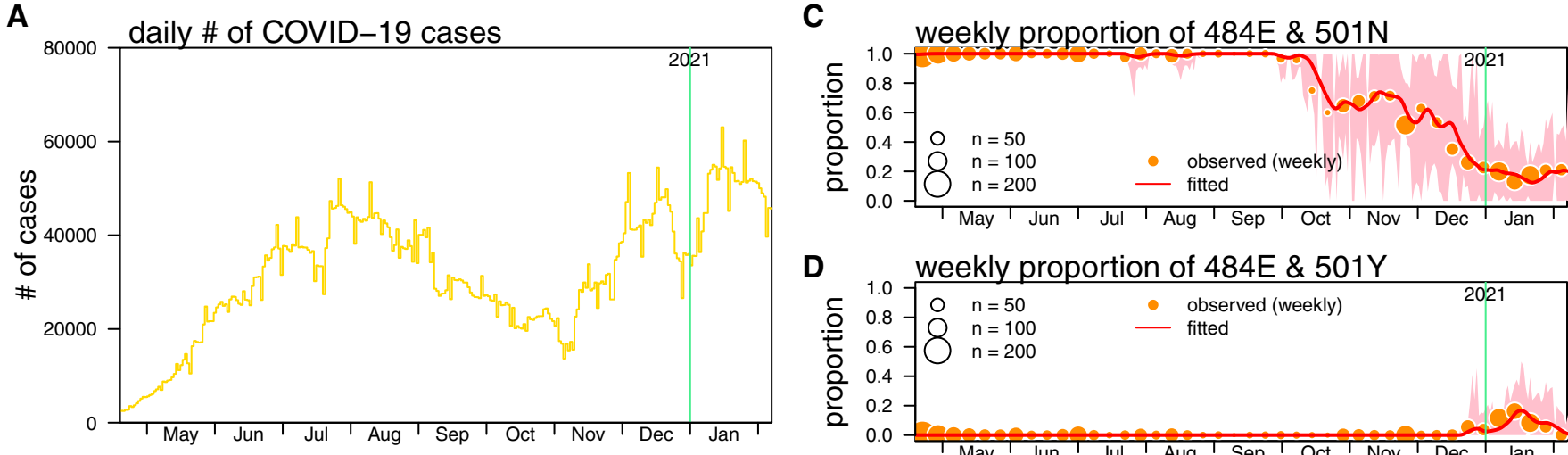

D

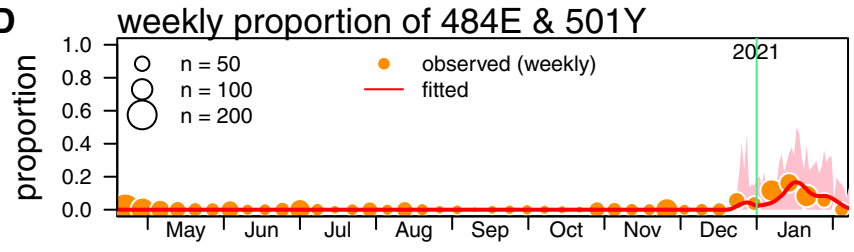

B

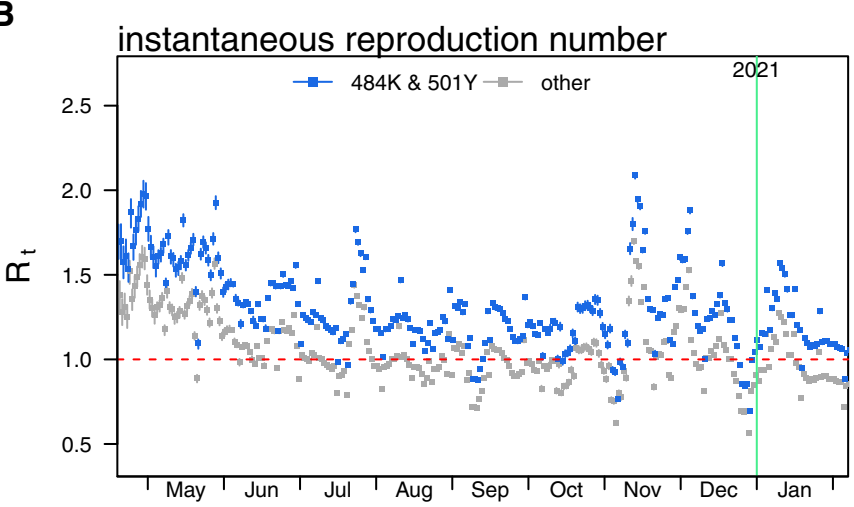

E
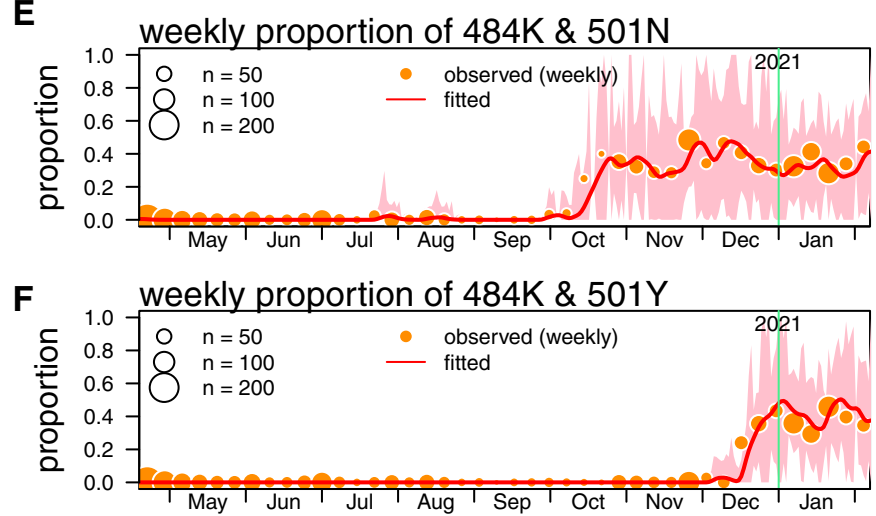

FIGURE 1. (A) The daily number of COVID-19 cases in Brazil, (B) the reconstructed reproduction number $\left(R_{t}\right)$, (C) proportions of the 484E/501N variants, (D) 484E/501Y variants (including B.1.1.7 or Alpha variants), (E) $484 \mathrm{~K} / 501 \mathrm{~N}$ variants (including P.2 or Zeta variants), and (F) $484 \mathrm{~K} / 501 \mathrm{Y}$ variants (including P.1 or Gamma variants). Panel B shows the estimated $R_{t} s$ of $484 \mathrm{~K} / 501 \mathrm{Y}$ variants (in blue) and the other three types of variants (in gray). The dots are the estimates, and bars are the $95 \%$ confidence intervals (Cls). In panels $\mathrm{C}$ through $\mathrm{F}$, the dots are the observations, the curve indicates the mean fitting results, and the shading area indicates the $95 \% \mathrm{Cl}$. This figure appears in color at www.ajtmh.org. 
consider the expected new incidences infected by $j$-th VoC at time $t$, denoted by $\mathbf{E}\left[C_{j}(t)\right]$, which can be modeled in Eq. (1).

$$
\mathbf{E}\left[C_{j}(t)\right]=R_{j}(t) \int_{0}^{\infty} w(\tau) \rho_{j}(t-\tau) C(t-\tau) \mathrm{d} \tau .
$$

Equation (1) will be used to formulate the likelihood function in the remaining sections. Note that the index $j$ is merely used for labeling instead of ranking.

Transmission advantages and their interactive effects. For convenience, we label the original variant as the $(j=)$ 0 -th VoC, that is, the $484 \mathrm{E} / 501 \mathrm{~N}$ variant, and thus its associated variant-specific reproduction number is $R_{j}=0$. Similarly, we label the $484 \mathrm{E} / 501 \mathrm{Y}, 484 \mathrm{~K} / 501 \mathrm{~N}$, and $484 \mathrm{~K} / 501 \mathrm{Y}$ variants as the $j=1,2$, and $3 \mathrm{VoC}$, respectively.

Following previous work, ${ }^{28,58}$ the transmission advantage of the mutated variant against the original type is defined as the ratio (denoted by $\eta$ ) of the strain-specified reproduction numbers. Considering the transmissibility of the original $484 \mathrm{E} / 501 \mathrm{~N}$ variant $\left(R_{j=0}\right)$ as the reference level, the transmission advantage of the $j$-th $\mathrm{VoC}$ is $\eta_{j}=\frac{R_{j}}{R_{j=0}}$. Thus, the reproduction number of cases infected by the $j$-th $\mathrm{VoC}$ is $R_{j}=\eta_{j} \cdot R_{j=0}$, and $\eta_{j=0}=1$ by definition. If $\eta_{j}>1$, the $j$-th variant may be more infectious than the original genetic variant, and vice versa. In addition, the overall reproduction number is $R_{j=0}(t) \sum_{j}\left[\eta_{j} \rho_{j}(t)\right]$.

We consider $\eta_{j}$ as a constant, which reflects an intrinsic nature of the $j$-th SARS-CoV-2 variant, and thus $\eta_{j}$ is invariant with time. Hence, we have $R_{j}(t)=\eta_{j} \cdot R_{j=0}(t)$ for all time $t$. Then, we calculate the expected number of COVID-19 cases, $\mathbf{E}[C(t)]$, at time $t$ in Eq. (2).

$$
\mathbf{E}[C(t)]=R_{j=0}(t) \sum_{j}\left[\eta_{j} \int_{0}^{\infty} w(\tau) \rho_{j}(t-\tau) C(t-\tau) \mathrm{d} \tau\right] .
$$

As such, for the observed sequencing data, the expected chance (or probability) that a randomly selected strain at the $t$-th day is $j$-th $\operatorname{VoC}, \mathrm{E}\left[\rho_{j}(t)\right]$, is given in Eq. (3).

$$
\mathbf{E}\left[\rho_{j}(t)\right]=\frac{\mathbf{E}\left[C_{j}(t)\right]}{\mathbf{E}[C(t)]}=\frac{\eta_{j} \int_{0}^{\infty} w(\tau) \rho_{j}(t-\tau) C(t-\tau) \mathrm{d} \tau}{\sum_{j}\left[\eta_{j} \int_{0}^{\infty} w(\tau) \rho_{j}(t-\tau) C(t-\tau) \mathrm{d} \tau\right]}
$$

Straightforwardly, $\sum_{j} \mathbf{E}\left[\rho_{j}(t)\right]=1$ for all $t$.

For the E484K and N501Y substitutions, the 484E/501Y $(j=1$, i.e., including the B.1.1.7 or Alpha variants) and $484 \mathrm{~K}$ / $501 \mathrm{~N}(j=2$, i.e., including the P.2 or Zeta variants) are two variants with merely one substitution, whereas the $484 \mathrm{~K} / 501 Y$ variant $(j=3$, i.e., including the P.1 or Gamma variants) has both substitutions. To explore the interactive effects on the variant-specific reproduction number, we compare $\eta_{j=3}$ and the product of $\left(\eta_{j=1} \cdot \eta_{j=2}\right)$. If $\eta_{j=3}$ is larger than $\left(\eta_{j=1} \cdot \eta_{j=2}\right)$, the E484K and N501Y substitutions may enhance the transmissibility than their separated partial effects, and vice versa.

Likelihood-based inference. According to Eq. (2), we construct the likelihood function $L_{t}^{(\mathrm{c})}$ of the daily number of cases using a Poisson-distributed framework with observation at $C_{t}$ and rate parameter at $\mathbf{E}\left[C_{t}\right]$ as in Eq. (4).

$$
L_{t}^{(\mathrm{c})}\left(C_{t} \mid \mathbf{E}\left[C_{t}\right]\right)=\frac{\mathbf{E}\left[C_{t}\right]^{C_{t}} \cdot e^{-\mathbf{E}\left[C_{t}\right]}}{C_{t} !}
$$

Here, the $C_{t}$ is the observed number of COVID-19 cases on day $t$ and is the discretized $C(t)$, which means $C_{t}=\int_{\text {day } t} C(x) \mathrm{d} x$. The value of $C_{t}$ can be obtained from the number of COVID-19 cases time series as shown in Figure $1 \mathrm{~A}$. Note that the superscript ${ }^{(\mathrm{c})}$ merely indicates that the likelihood function is for the number of cases, which does not indicate the power.

For the observed sequencing data, we denote the numbers of $j=0,1,2$, and 3 variants by $m_{j=0, t}, m_{j=1, t}, m_{j=2, t}$, and $m_{j=3, t}$, respectively, for the day $t$. Thus, we model the sampling process of genetic variants using a generalized Bernoulli distribution, that is, categorical distribution, with probabilities at $\mathbf{E}\left[\rho_{j}(t)\right] \mathbf{s}$ in Eq. (3). The likelihood function $L_{t}^{(\mathrm{s})}$ is constructed in Eq. (5).

$$
L_{t}^{(s)}\left(m_{j=0, t}, \ldots, m_{j=3, t} \mid \mathbf{E}\left[\rho_{j=0, t}\right], \ldots, \mathbf{E}\left[\rho_{j=3, t}\right]\right)=\prod_{j} \mathbf{E}\left[\rho_{j, t}\right]^{m_{j, t}} .
$$

Here, the $\mathbf{E}\left[\rho_{j, t}\right]$ is the expectation of variant prevalence for day $t$. Note that the superscript ${ }^{(\mathrm{s})}$ merely indicates that the likelihood function is for genetic variants, which does not indicate the power.

With Eqs. (4) and (5), we reconstruct the $R_{j=0, t}$ time series, denoted by $\left\{R_{j=0, t}\right\}$, and estimate $\eta_{j=1}, \eta_{j=2}$, and $\eta_{j=3}$ using the overall log-likelihood function $\ell$ defined in Eq. (6).

$$
\begin{gathered}
\ell\left(\left\{R_{j=0, t}\right\}, \eta_{j=1}, \eta_{j=2}, \eta_{j=3} \mid\left\{C_{t}\right\},\left\{m_{j=0, t}\right\},\right. \\
\left.\ldots,\left\{m_{j=3, t}\right\}\right)=\sum_{t} \log \left[L_{t}^{(c)} \times L_{t}^{(s)}\right] .
\end{gathered}
$$

We calculate the maximum likelihood estimates (MLE) of parameters to determine transmission advantage of 484E/ $501 \mathrm{Y}\left(\eta_{j=1}\right), 484 \mathrm{~K} / 501 \mathrm{~N}\left(\eta_{j=2}\right)$, and $484 \mathrm{~K} / 501 \mathrm{Y}\left(\eta_{j=3}\right)$ variants by using the likelihood framework defined in Eq. (6). The 95\% confidence intervals $(95 \% \mathrm{Cl})$ are calculated using the profile likelihood estimation framework with a $\chi^{2}$ quantile as the cutoff, ${ }^{59,60}$ which has also been adopted in previous work. ${ }^{61-67}$

Fitting schemes and their selection. We explore the interactive effects between E484K and N501Y substitutions of SARS-CoV-2 in shaping the transmission advantage in Brazil. We consider the following eight fitting scenarios with respect to $\eta_{j}$ and investigate the role of E484K and N501Y substitutions contributing to the transmissibility of COVID-19:

- all of $\eta_{j=1}, \eta_{j=2}$, and $\eta_{j=3}$ are assumed at 1 : scenario (\#1): $\eta_{j=1}=\eta_{j=2}=\eta_{j=3}=1$;

- two of $\eta_{j=1}, \eta_{j=2}$, or $\eta_{j=3}$ are assumed at 1 , and the remaining one is freely estimated:

scenario (\#2): $\eta_{j=1}=\eta_{j=2}=1$,

scenario (\#3): $\eta_{j=1}=\eta_{j=3}=1$, and

scenario (\#4): $\eta_{j=2}=\eta_{j=3}=1$;

- one of $\eta_{j=1}, \eta_{j=2}$, or $\eta_{j=3}$ is assumed at 1 , and the remaining two are freely estimated:

scenario (\#5): $\eta_{j=1}=1$,

scenario (\#6): $\eta_{j=2}=1$, and

scenario (\#7): $\eta_{j=3}=1$; 
TABLE 1

The summary of transmission advantage estimates of different types of SARS-CoV-2 variants in Brazil under different scenarios

\begin{tabular}{|c|c|c|c|c|c|c|c|c|c|}
\hline \multirow[b]{3}{*}{ Scenario } & \multicolumn{4}{|c|}{ Transmission advantage of } & \multirow[b]{3}{*}{ AIC } & \multirow[b]{3}{*}{$\mathrm{AlCc}$} & \multirow[b]{3}{*}{$\mathrm{BIC}$} & \multirow[b]{3}{*}{ HQIC } & \multirow[b]{3}{*}{ Remarks } \\
\hline & \multirow{2}{*}{$\frac{\text { Original variant }}{484 \mathrm{E} / 501 \mathrm{~N}}$} & \multicolumn{3}{|c|}{ New emerging variant } & & & & & \\
\hline & & 484E/501Y & $484 \mathrm{~K} / 501 \mathrm{~N}$ & $484 \mathrm{~K} / 501 \mathrm{Y}$ & & & & & \\
\hline (\#1) & 1 (reference) & 1 (assumed) & 1 (assumed) & 1 (assumed) & 6895.6 & 6960.3 & 8915.2 & 7615.1 & Baseline model \\
\hline (\#2) & 1 (reference) & 1 (assumed) & 1 (assumed) & $1.23(1.04-1.41)$ & 6888.7 & 6953.8 & 8914.4 & 7610.4 & Two types of new \\
\hline (\#3) & 1 (reference) & 1 (assumed) & $1.05(0.93-1.17)$ & 1 (assumed) & 6893.5 & 6958.6 & 8919.3 & 7615.2 & variant are assumed \\
\hline (\#4) & 1 (reference) & $1.08(0.78-1.41)$ & 1 (assumed) & 1 (assumed) & 6893.5 & 6958.6 & 8919.3 & 7615.2 & to have no effect \\
\hline (\#5) & 1 (reference) & 1 (assumed) & $1.12(1.01-1.22)$ & $1.28(1.08-1.47)$ & 6888.3 & 6953.8 & 8920.3 & 7612.2 & One type of new \\
\hline (\#6) & 1 (reference) & $1.15(0.83-1.52)$ & 1 (assumed) & $1.21(1.05-1.42)$ & 6889.9 & 6955.4 & 8921.9 & 7613.8 & variant is assumed \\
\hline$(\# 7)$ & 1 (reference) & $1.08(0.79-1.43)$ & $1.04(0.92-1.16)$ & 1 (assumed) & 6895.3 & 6960.8 & 8927.2 & 7619.2 & to have no effect \\
\hline (\#8) & 1 (reference) & $1.26(0.92-1.66)$ & $1.14(1.03-1.27)$ & $1.33(1.13-1.56)$ & 6888.4 & 6954.3 & 8926.5 & 7614.5 & Full model \\
\hline
\end{tabular}
selected as the main result, and shown in Figure 1.

- none of $\eta_{j=1}, \eta_{j=2}$, or $\eta_{j=3}$ is assumed at 1 , and all three of them are freely estimated:

scenario (\#8): none of $\eta_{j=1}, \eta_{j=2}$, or $\eta_{j=3}$ is assumed at 1 .

The settings of the eight fitting scenarios are presented in Table 1.

We conduct the model fitting and parameter estimation under each scenario. The scenario with the best fitting performance is selected according to lowest values of 4 different information criteria including Akaike information criterion (AIC), corrected AIC for small sample size (AICc), Bayesian information criterion (BIC), and Hannan-Quinn information criterion (HQIC).

Sensitivity analysis. Sensitivity analysis was conducted to examine the robustness and significance of the determine transmission advantage estimates, that is, $\eta s$. We examine the consistency of both directions of the effects and their $95 \%$ confidence intervals $(\mathrm{Cl})$ under alternative settings. The following three sensitivity checking schemes are performed.

For the first scheme, we consider a univariate logistic regression model between the overall $R_{t}$ as response and $\rho_{j=1, t}, \rho_{j=2, t}$, and $\rho_{j=3, t}$ as regressors. The regression coefficients of all $\rho_{j, t}$ are evaluated as the effect size of SARSCoV2 variants on the overall transmissibility of COVID-19. For the second scheme, we repeat the estimating process of transmission advantage with alternative PDF of GT, that is, $w(\cdot)$, which is introduced in the previous section, "Variantspecific reproduction number." We consider shorter and longer versions of mean GT at 4 days, ${ }^{53,68}$ and 7.5 days, ${ }^{2}$ respectively. For the third scheme, we repeat the analysis by replacing the Poisson-distributed likelihood function in Eq. (4) with a negative binomial (NB) distribution to further account for the superspreading potential of COVID-19 transmission. For the setting of NB distribution, we fixed the dispersion parameter at 0.4 , which follows the estimation in recent studies. ${ }^{55,69-74}$

\section{RESULTS AND DISCUSSION}

As of this writing, in Brazil, the epidemic curve had grown since March 2020 with two major epidemic waves, the first in August 2020, and the second is ongoing (Figure 1A). The original $484 \mathrm{E} / 501 \mathrm{~N}$ variants started being replaced by the other three types of variants in October 2020 and had almost vanished in Brazil after January 2021 (Figure 1C). In January 2021 , the prevalence of the emerging $484 \mathrm{~K}$ variants was
$71.1 \%$ and $46.6 \%$ for $501 Y$ variants. The prevalence of $484 \mathrm{E} / 501 \mathrm{Y}(j=1$ type) variants is $10.1 \%, 34.6 \%$ for $484 \mathrm{~K} /$ $501 \mathrm{~N}(j=2$ type) variants, and $36.5 \%$ for $484 \mathrm{~K} / 501 \mathrm{Y}(j=3$ type) variants (Figure 1D-F). As such, the linkage disequilibrium (LD) is calculated at 0.03 , which indicates the occurrence of two mutations is likely random. Specially, the 484K/ $501 Y$ variants are classified into the B.1.1.28.1 (or P.1, or 20J/501Y.V3) lineage.

For the eight fitting scenarios summarized in Table 1, we find that the transmission advantage of $484 \mathrm{~K} / 501 \mathrm{Y}\left(\eta_{j=3}\right)$ variants is estimated larger than 1 significantly and consistently. In contrast, the scale of $\eta_{j=1}$ for $484 \mathrm{E} / 501 \mathrm{Y}$ variants appears statistically unclear compared with 1 , that is, not significantly larger than 1 . For the model selection, scenario 2 has the lowest AICc, BIC, and HQIC, and scenario 5 has the lowest $\mathrm{AIC}$, and AICc. Both scenarios 2 and 5 also have close values of AIC with a difference of only 0.4. As such, scenario 2 is considered the main result and thus is presented in Figure 1B.

The modeling framework in this study links the mutation activity at molecular scale and COVID-19 transmissibility at population scale. We reconstruct the instantaneous reproduction numbers $\left(R_{t}\right)$ of COVID-19 cases infected by $484 \mathrm{~K} / 501 \mathrm{Y}$ variants and other variants in Brazil under fitting scenario 2 (Figure 1B). The overall trends of reproduction numbers are relatively high in the early phase of outbreak before and in May 2020 and the November 2020 for the second major epidemic wave, but gradually decrease thereafter. The average scale of reproduction number during the early outbreak is largely consistent with previous estimates. ${ }^{2,3,75-77}$

We report the estimated proportions of four types of SARS-CoV-2 variants, $\mathbf{E}\left[\rho_{t}\right]$, fit the observed sequencing data well (Figure 1C-F). We infer the transmission advantage $\eta_{j=3}$ for $484 \mathrm{~K} / 501 \mathrm{Y}$ variants at $1.23(95 \% \mathrm{Cl}: 1.04-1.41)$, which means the E484K and N501Y substitutions together increase $23 \%$ of COVID-19's transmissibility in Brazil, whereas other emerging variants, $484 \mathrm{E} / 501 \mathrm{Y}$ and $484 \mathrm{~K} / 501 \mathrm{~N}$, are unlikely to have significant transmission advantage. Thus, in Figure $1 \mathrm{~B}$, the reproduction number of the $484 \mathrm{~K} / 501 \mathrm{Y}$ variant appears higher than that of the other genotypes (non-484K/ $501 Y$, or non-Gamma variants). For sensitivity checking, we find that the $\eta_{j=3}$ estimates are consistently and significantly larger than 1 in similar scales as the main estimates (data not shown), which validates our findings.

We focus on the second major epidemic wave because E484K and N501Y substitutions emerged during the same 
period. Although the reproduction number of non-484K/ $501 Y$ variants has fluctuated around 1 since December 2020 , the reproduction number, $R_{j=3}$, of $484 \mathrm{~K} / 501 \mathrm{Y}$ variants are largely greater than 1 during the same period, which has led to a large epidemic wave in Brazil in 2021 (see Figure $1 \mathrm{~A})$. Given that $484 \mathrm{~K} / 501 \mathrm{Y}$ variants trend to reach fixation, both the herd immunity threshold and intrinsic growth rate of epidemic may increase, which was previously discussed regarding the situation in United Kingdom, ${ }^{12,25}$ and thus the local nonpharmaceutical interventions for COVID19 control may be enforced. Hence, we highlight the importance of our analytical framework, such that the public health risks related to viral mutations may be controllable with early preparedness.

The increase in transmissibility associated with the 484K/ $501 Y$ variants is biologically reasonable. The N501Y substitution is a mutation on a key contact residue in the receptor binding domain on the $S$ protein $^{78}$ and is found to increase the ability of human angiotensin-converting enzyme 2 binding and cell infectivity in animal models, ${ }^{79}$ which appears similar to the previous D614G substitution. ${ }^{80}$ The E484K substitution is also located in the viral RBD and confers resistance to several monoclonal antibodies by affecting the binding process. ${ }^{6,81,82}$ However, according to the $\eta_{j=1}$ estimates in Table 1, N501Y substitution alone appears less likely to form an advantage in fitness without accompanying 484K. Similarly, E484K substitution alone also might not secure a concrete transmission advantage robustly or significantly. Our finding indicates that the combined impact of $484 \mathrm{~K} / 501 \mathrm{Y}$ outperforms their independent effects, which implies statistically an interactive relationship.

Previous studies reported a higher case fatality risk among the individuals infected by SARS-CoV-2 strains in the B.1.1.7 lineage, ${ }^{42,83}$ some of which carry both E484K and N501Y substitutions. Given the transmission advantage of $484 \mathrm{~K} / 501 \mathrm{Y}$ (i.e., $\eta_{j=3}>1$ ), the increasing intensity of COVID19-related mortality is a public health concern. Clinical severity remains largely unassessed for the B.1.1.28 lineage in Brazil, and unexpected clinical outcomes may warrant adjustments in the treatment strategies. The emergence of $484 \mathrm{~K} / 501 \mathrm{Y}$ variants and its mutations (e.g., K417N or V1176F), combined with other VoC in Brazil and other places, implies the capacity of SARS-CoV-2 to evolve new phenotypes rapidly. ${ }^{84}$ Although the neutralizing level of BNT162b2 vaccine-elicited sera is recently found to be satisfactory for $484 \mathrm{~K} / 501 \mathrm{Y}$ variants, ${ }^{20}$ further investigation is required for other vaccine candidates at population scale.

This study has the following limitations. First, our analysis was based on the sequence data released in GISAID and thus is subject to the selection bias of sequences being released to the public domine. ${ }^{12}$ Second, the reconstruction of reproduction numbers relies on the setting of the generation time (GT). Theoretically, the GT distribution might be altered by the mutated strains. However, by screening the literature, we find no evidence that GT is associated with the E484K or N501Y substitution in SARS-CoV-2, and thus we model GT distribution of COVID-19 $w(\cdot)$ as a fixed Gamma distribution, following previous studies. ${ }^{50-54}$ Third, we consider $w(\cdot)$ as a fixed distribution. In the real-world situation, the time interval between transmission generations might vary, ${ }^{75,85}$ which may affect the reconstruction of the reproduction number. However, the long-term trends of $R_{t}$ estimates are unlikely to change due to slight variation in GT. ${ }^{75,86}$ Thus, we consider the impact of this limitation on the inference of transmission advantage may be negligible, and our model can be extended to a more complex context with the time-varying GT data available. Fourth, due to the lack of data from different Brazilian regions, we aggregated the national COVID-19 cases in Brazil to reconstruct the reproduction number series (Figure 1B). We acknowledge this analytical scheme neglects the heterogeneities in epidemiological characteristics of COVID-19 transmission, ${ }^{55,74,87,88}$ geographic separation in SARS-CoV-2 variants, ${ }^{89}$ individual response and vulnerability to COVID-19, ${ }^{90-92}$ and various nonpharmaceutical interventions ${ }^{93,94}$ for wide regions across different Brazilian locations. We note that the transmission advantage may vary under different local settings or situations. Fifth, ideally, $C(t)$ in the $R_{t}$ estimation should be the number of COVID19 cases with onset at time $t$. However, because surveillance data by date of onset are unavailable, we adapted the current dataset by reporting data as a proxy for the COVID-19 incidence time series. If one considers a constant reporting lag, the $R_{t}$ estimates will have the same trends but are shifted for this lag. Considering that a similar reporting delay also occurred during the collection of SARS-CoV-2 sequencing data, the effects of the two reporting lags could be counteracted. We believe that this approximation is unlikely to affect the main conclusions of this study. Furthermore, with detailed reporting lag of information for each individual case, adjustments for reporting delay can be carried out based on our current analytical framework. Sixth, this study focuses on exploring the effects on changing disease transmissibility associated with mutation activities, but real-world biological mechanisms, which are usually more complex, remain uncovered. Future studies are needed to explore the mechanisms of how E484K and N501Y mutations in SARS-CoV-2 affect the transmissibility of COVID-19. Seventh, there exist other mutations in the B.1.1.28 lineage, such as $\mathrm{K} 417 \mathrm{~N}$ and V1176F, but we merely considered the E484K and N501Y because they are dominant in B.1.1.28.1 and B.1.1.28.2 lineages, respectively. Given the lack of individual patient information, time-series data were used in this work, which means there is information loss from the data aggregation. As pointed out in previous studies, ${ }^{83}$ the independent effects of each commutation may not be disentangled in this study due to identification issues that the samples might fail to inform each estimate. Eighth, the interpretation of our findings should be limited to the COVID-19 epidemics in Brazil, but similar investigations could be conducted for other regions. Ninth, for simplification, we consider the transmission advantage $(\eta)$ of new variants versus the wildtype as constant over time. This model assumption may not necessarily be strictly held considering several real-world determinants, including the accumulation of population immunity against different strains, ${ }^{24}$ selection pressure due to intervention strategies, ${ }^{27,58}$ and behavioral factors related to disease spread and transmission. ${ }^{95}$ Alternatively, the estimating framework of $\eta$ can be extended into a real-time basis. Lastly, as a data-driven study, the estimated association should be treated with caution. In an ecological setting, ${ }^{13}$ although our analysis provides 
statistical evidence on the likelihood of causality, the findings in this study cannot guarantee causality, which requires further biomedical experiments for verification.

Received April 15, 2021. Accepted for publication August 31, 2021.

Published online September 27, 2021.

Note: Supplemental file appears at www.ajtmh.org.

Acknowledgments: The SARS-CoV-2 protein sequences were retrieved from the global initiative on sharing all influenza data (GISAID; http://platform.gisaid.org). The contributions of the submitting and the originating laboratories were acknowledged. This study is conducted using the resources of Alibaba Cloud Intelligence High Performance Cluster computing facilities, which is free of charge for COVID-19 research. The authors thank Ms. J. Lou for helping collect the sequences data, and Ms. L. Cao and Dr. M. H. Wang for a brief discussions on the preliminary results. The American Society of Tropical Medicine and Hygiene has waived the Open Access fee for this article due to the ongoing COVID-19 pandemic.

Financial support: J. R. was supported by SJTU Initiation Program for New Youth Teachers (No. 21X010501093).

Authors' addresses: Shi Zhao, JC School of Public Health and Primary Care, Chinese University of Hong Kong, Hong Kong, China, and CUHK Shenzhen Research Institute, Shenzhen, China, E-mail: zhaoshi.cmsa@gmail.com. Jinjun Ran, School of Public Health, Shanghai Jiao Tong University School of Medicine, Shanghai, China, E-mail: jinjunr@sjtu.edu.cn. Lefei Han, School of Global Health, Chinese Center for Tropical Diseases Research, Shanghai Jiao Tong University School of Medicine, Shanghai, China, E-mail: Ifhan@sjtu. edu.cn.

This is an open-access article distributed under the terms of the Creative Commons Attribution (CC-BY) License, which permits unrestricted use, distribution, and reproduction in any medium, provided the original author and source are credited.

\section{REFERENCES}

1. Hu B, Guo H, Zhou P, Shi ZL, 2021. Characteristics of SARSCoV-2 and COVID-19. Nat Rev Microbiol 19: 141-154.

2. Li $Q$ et al., 2020. Early transmission dynamics in Wuhan, China, of novel coronavirus-infected pneumonia. N Engl J Med 382: 1199-1207.

3. Wu JT, Leung K, Leung GM, 2020. Nowcasting and forecasting the potential domestic and international spread of the 2019nCoV outbreak originating in Wuhan, China: a modelling study. Lancet 395: 689-697.

4. Kutter JS, Spronken MI, Fraaij PL, Fouchier RA, Herfst S, 2018. Transmission routes of respiratory viruses among humans. Curr Opin Virol 28: 142-151.

5. Fraser C, Riley S, Anderson RM, Ferguson NM, 2004. Factors that make an infectious disease outbreak controllable. Proc Natl Acad Sci USA 101: 6146-6151.

6. Baum A et al., 2020. Antibody cocktail to SARS-CoV-2 spike protein prevents rapid mutational escape seen with individual antibodies. Science 369: 1014-1018.

7. Tsetsarkin KA, Vanlandingham DL, McGee CE, Higgs S, 2007. A single mutation in chikungunya virus affects vector specificity and epidemic potential. PLoS Pathog 3: e201.

8. Tang JW, Tambyah PA, Hui DS, 2021. Emergence of a new SARS-CoV-2 variant in the UK. J Infect 82: e27-e28.

9. Tang JW, Toovey OTR, Harvey KN, Hui DDS, 2021. Introduction of the South African SARS-CoV-2 variant 501Y. V2 into the UK. J Infect 82: e8-e10.

10. Claro IM et al., 2021. Local Transmission of SARS-CoV-2 lineage B.1.1.7, Brazil, December 2020. Emerg Infect Dis 27: 970972.

11. Galloway SE et al., 2021. Emergence of SARS-CoV-2 b. 1.1. 7 lineage-United States, December 29, 2020-January 12, 2021. Morb Mortal Wkly Rep 70: 95.

12. Leung K, Shum MH, Leung GM, Lam TT, Wu JT, 2021. Early transmissibility assessment of the N501Y mutant strains of
SARS-CoV-2 in the United Kingdom, October to November 2020. Euro Surveill 26: 2002106.

13. Graham MS et al., 2021. Changes in symptomatology, reinfection, and transmissibility associated with the SARS-CoV-2 variant B.1.1.7: an ecological study. The Lancet Public Health 6: e335-e345.

14. Public Health England, 2021. Variants: Distribution of Cases Data: Variants of Concern or Under Investigation: Data up to 7 April 2021. Available at: https://www.gov.uk/government/ publications/covid-19-variants-genomically-confirmed-casenumbers/variants-distribution-of-cases-data.

15. Toovey OTR, Harvey KN, Bird PW, Tang JW-TW-T, 2021. Introduction of Brazilian SARS-CoV-2 484K. V2 related variants into the UK. J Infect 82: e23-e24.

16. Voloch CM et al., 2021. Genomic characterization of a novel SARS-CoV-2 lineage from Rio de Janeiro, Brazil. J Virol 95: e00119-e21.

17. Wise J, 2021. COVID-19: the E484K mutation and the risks it poses. BMJ 372: n359.

18. Walensky RP, Walke HT, Fauci AS, 2021. SARS-CoV-2 variants of concern in the United States-challenges and opportunities. JAMA 325: 1037-1038.

19. Rondinone $V$ et al., 2021. VOC 202012/01 variant is effectively neutralized by antibodies produced by patients infected before its diffusion in Italy. Viruses-Basel 13: 276.

20. Xie XP et al., 2021. Neutralization of SARS-CoV-2 spike 69/70 deletion, E484K and N501Y variants by BNT162b2 vaccineelicited sera. Nat Med 27: 620-621.

21. Moore JP, Offit PA, 2021. SARS-CoV-2 vaccines and the growing threat of viral variants. JAMA 325: 821-822.

22. Muik A et al., 2021. Neutralization of SARS-CoV-2 lineage B. 1.1. 7 pseudovirus by BNT162b2 vaccine-elicited human sera. Science 371: 1152-1153.

23. Supasa $P$ et al., 2021. Reduced neutralization of SARS-CoV-2 B. 1.1. 7 variant by convalescent and vaccine sera. Cell 184: 2201-2211.

24. Faria NR et al., 2021. Genomics and epidemiology of the P. 1 SARS-CoV-2 lineage in Manaus, Brazil. Science 372: 815-821.

25. Zhao $S$ et al., 2021. Quantifying the transmission advantage associated with N501Y substitution of SARS-CoV-2 in the UK: an early data-driven analysis. J Travel Med 28: taab011.

26. Davies NG et al., 2021. Estimated transmissibility and impact of SARS-CoV-2 lineage B.1.1.7 in England. Science 372: eabg3055.

27. Volz E et al., 2021. Assessing transmissibility of SARS-CoV-2 lineage B. 1.1. 7 in England. Nature 593: 266-269.

28. Volz $E$ et al., 2021. Evaluating the effects of SARS-CoV-2 spike mutation D614G on transmissibility and pathogenicity. Cell 184: 64-75 e11.

29. Hou YJ et al., 2020. SARS-CoV-2 D614G variant exhibits efficient replication ex vivo and transmission in vivo. Science 370: 1464-1468.

30. Zhang $L$ et al., 2020. SARS-CoV-2 spike-protein D614G mutation increases virion spike density and infectivity. Nat Commun 11: 6013 .

31. Zhao $S$ et al., 2021. Modelling the association between COVID19 transmissibility and D614G substitution in SARS-CoV-2 spike protein: using the surveillance data in California as an example. Theor Biol Med Model 8: 10.

32. da Silva Francisco R Jr et al., 2021. Pervasive transmission of E484K and emergence of VUI-NP13L with evidence of SARSCoV-2 co-infection events by two different lineages in Rio Grande do Sul, Brazil. Virus Res 296: 198345.

33. Khan A, Zia T, Suleman M, Khan T, Ali SS, Abbasi AA, Mohammad A, Wei DQ, 2021. Higher infectivity of the SARS-CoV-2 new variants is associated with $\mathrm{K} 417 \mathrm{~N} / \mathrm{T}$, E484K, and N501Y mutants: an insight from structural data. J Cell Physiol 236: 7045-7057.

34. Hie B, Zhong ED, Berger B, Bryson B, 2021. Learning the language of viral evolution and escape. Science 371: 284-288.

35. Gog JR, Rimmelzwaan GF, Osterhaus ADME, Grenfell BT, 2003. Population dynamics of rapid fixation in cytotoxic $T$ lymphocyte escape mutants of influenza A. Proc Natl Acad Sci USA 100: 11143-11147. 
36. Smith DJ, Lapedes AS, de Jong JC, Bestebroer TM, Rimmelzwaan GF, Osterhaus AD, Fouchier RA, 2004. Mapping the antigenic and genetic evolution of influenza virus. Science 305: 371-376.

37. Zhao S, Lou J, Cao L, Chen Z, Chan RW, Chong MK, Zee BC, Chan PK, Wang MH, 2020. Quantifying the importance of the key sites on haemagglutinin in determining the selection advantage of influenza virus: using $\mathrm{A} / \mathrm{H} 3 \mathrm{~N} 2$ as an example. $J$ Infect 81: 452-482.

38. Berkhoff EGM, Boon ACM, Nieuwkoop NJ, Fouchier RAM, Sintnicolaas K, Osterhaus A, Rimmelzwaan GF, 2004. A mutation in the HLA-B* 2705-restricted NP383-391 epitope affects the human influenza A virus-specific cytotoxic T-lymphocyte response in vitro. J Virol 78: 5216-5222.

39. Rimmelzwaan GF, Berkhoff EGM, Nieuwkoop NJ, Fouchier RAM, Osterhaus A, 2004. Functional compensation of a detrimental amino acid substitution in a cytotoxic-T-lymphocyte epitope of influenza a viruses by comutations. J Virol 78: 8946-8949.

40. Rimmelzwaan GF, Berkhoff EGM, Nieuwkoop NJ, Smith DJ, Fouchier RAM, Osterhaus A, 2005. Full restoration of viral fitness by multiple compensatory co-mutations in the nucleoprotein of influenza A virus cytotoxic T-lymphocyte escape mutants. J Gen Virol 86: 1801-1805.

41. Ferguson NM, Cummings DA, Cauchemez S, Fraser C, Riley S, Meeyai A, lamsirithaworn S, Burke DS, 2005. Strategies for containing an emerging influenza pandemic in Southeast Asia. Nature 437: 209-214.

42. Davies NG et al., 2021. Increased mortality in community-tested cases of SARS-CoV-2 lineage B.1.1.7. Nature 593: 270-274.

43. Shu Y, McCauley J, 2017. GISAID: global initiative on sharing all influenza data-from vision to reality. Euro Surveill 22: 30494.

44. Katoh K, Rozewicki J, Yamada KD, 2019. MAFFT online service: multiple sequence alignment, interactive sequence choice and visualization. Brief Bioinform 20: 1160-1166.

45. World Health Organization, 2021. Coronavirus Disease 2019 (COVID-19) Situation Reports. Geneva, Switzerland: World Health Organization. Available at: https://www.who.int/ emergencies/diseases/novel-coronavirus-2019/situationreports.

46. Cori A, Ferguson NM, Fraser C, Cauchemez S, 2013. A new framework and software to estimate time-varying reproduction numbers during epidemics. Am J Epidemiol 178: 15051512

47. Zhao S, Musa SS, Hebert JT, Cao P, Ran J, Meng J, He D, Qin $\mathrm{J}$, 2020. Modelling the effective reproduction number of vector-borne diseases: the yellow fever outbreak in Luanda, Angola 2015-2016 as an example. PeerJ 8: e8601.

48. Champredon D, Dushoff J, Earn DJD, 2018. Equivalence of the Erlang-distributed SEIR epidemic model and the renewal equation. SIAM J Appl Math 78: 3258-3278.

49. Wallinga J, Lipsitch $M, 2007$. How generation intervals shape the relationship between growth rates and reproductive numbers. Proc Biol Sci 274: 599-604.

50. Ferretti L, Wymant C, Kendall M, Zhao L, Nurtay A, AbelerDorner L, Parker M, Bonsall D, Fraser C, 2020. Quantifying SARS-CoV-2 transmission suggests epidemic control with digital contact tracing. Science 368: eabb6936.

51. Ganyani T, Kremer C, Chen D, Torneri A, Faes C, Wallinga J, Hens N, 2020. Estimating the generation interval for coronavirus disease (COVID-19) based on symptom onset data, March 2020. Euro Surveill 25: 2000257.

52. Tindale LC et al., 2020. Evidence for transmission of COVID-19 prior to symptom onset. eLife 9: e57149.

53. Zhao S, 2020. Estimating the time interval between transmission generations when negative values occur in the serial interval data: using COVID-19 as an example. Math Biosci Eng 17: 3512-3519.

54. He X et al., 2020. Temporal dynamics in viral shedding and transmissibility of COVID-19. Nat Med 26: 672-675.

55. Adam DC, Wu P, Wong JY, Lau EHY, Tsang TK, Cauchemez S, Leung GM, Cowling BJ, 2020. Clustering and superspreading potential of SARS-CoV-2 infections in Hong Kong. Nat Med 26: $1714-1719$.
56. Biggerstaff $M$ et al., 2020. Early Insights from statistical and mathematical modeling of key epidemiologic parameters of COVID-19. Emerg Infect Dis J 26.

57. Zhao $S$ et al., 2021. Estimating the generation interval and inferring the latent period of COVID-19 from the contact tracing data. Epidemics 36: 100482.

58. Leung K, Lipsitch M, Yuen KY, Wu JT, 2017. Monitoring the fitness of antiviral-resistant influenza strains during an epidemic: a mathematical modelling study. Lancet Infect Dis 17: 339-347.

59. Fan JQ, Huang T, 2005. Profile likelihood inferences on semiparametric varying-coefficient partially linear models. Bernoulli 11: 1031-1057.

60. Bolker BM, 2008. Ecological Models and Data in R. Princeton, $\mathrm{NJ}$ : Princeton University Press.

61. Breto C, He DH, lonides EL, King AA, 2009. Time series analysis via mechanistic models. Ann Appl Stat 3: 319-348.

62. He D, lonides EL, King AA, 2010. Plug-and-play inference for disease dynamics: measles in large and small populations as a case study. J $R$ Soc Interface 7: 271-283.

63. Lin Q, Chiu AP, Zhao S, He D, 2018. Modeling the spread of Middle East respiratory syndrome coronavirus in Saudi Arabia. Stat Methods Med Res 27: 1968-1978.

64. Zhao $S$ et al., 2020. Estimating the serial interval of the novel coronavirus disease (COVID-19): a statistical analysis using the public data in Hong Kong from January 16 to February 15, 2020. Front Phys 8: 347.

65. Zhao S, Lou Y, Chiu APY, He D, 2018. Modelling the skip-andresurgence of Japanese encephalitis epidemics in Hong Kong. J Theor Biol 454: 1-10.

66. Wang $\mathrm{K}$ et al., 2020. Estimating the serial interval of the novel coronavirus disease (COVID-19) based on the public surveillance data in Shenzhen, China, from 19 January to 22 February 2020. Transbound Emerg Dis 67: 2818-2822.

67. Zhao $S$ et al., 2021. An early assessment of a case fatality risk associated with P.1 SARS-CoV-2 lineage in Brazil: an ecological study. J Travel Med (In press). doi: 10.1093/jtm/taab078.

68. Du Z, Xu X, Wu Y, Wang L, Cowling BJ, Meyers LA, 2020. Serial interval of COVID-19 among publicly reported confirmed cases. Emerg Infect Dis 26: 1341-1343.

69. Bi $Q$ et al., 2020. Epidemiology and transmission of COVID-19 in 391 cases and 1286 of their close contacts in Shenzhen, China: a retrospective cohort study. Lancet Infect Dis 20: 911-919.

70. Endo A, Abbott S, Kucharski AJ, Funk S, 2020. Estimating the overdispersion in COVID-19 transmission using outbreak sizes outside China. Wellcome Open Res 5: 67.

71. Lau MSY, Grenfell B, Thomas M, Bryan M, Nelson K, Lopman B, 2020. Characterizing superspreading events and age-specific infectiousness of SARS-CoV-2 transmission in Georgia, USA. Proc Natl Acad Sci USA 117: 22430-22435.

72. Tariq A, Lee Y, Roosa K, Blumberg S, Yan P, Ma S, Chowell G, 2020. Real-time monitoring the transmission potential of COVID-19 in Singapore, March 2020. BMC Med 18: 166.

73. Zhang Y, Li Y, Wang L, Li M, Zhou X, 2020. Evaluating transmission heterogeneity and super-spreading event of COVID-19 in a metropolis of China. Int $J$ Environ Res Public Health 17: 3705.

74. Zhao $S$ et al., 2021. Inferencing superspreading potential using zero-truncated negative binomial model: exemplification with COVID-19. BMC Med Res Methodol 21: 30

75. Ali ST, Wang L, Lau EHY, Xu XK, Du Z, Wu Y, Leung GM, Cowling BJ, 2020. Serial interval of SARS-CoV-2 was shortened over time by nonpharmaceutical interventions. Science 369: 1106-1109.

76. Chinazzi $\mathrm{M}$ et al., 2020. The effect of travel restrictions on the spread of the 2019 novel coronavirus (COVID-19) outbreak. Science 368: 395-400.

77. Gatto M, Bertuzzo E, Mari L, Miccoli S, Carraro L, Casagrandi R, Rinaldo A, 2020. Spread and dynamics of the COVID-19 epidemic in Italy: effects of emergency containment measures. Proc Natl Acad Sci USA 117: 10484-10491.

78. Starr TN et al., 2020. Deep mutational scanning of SARS-CoV-2 receptor binding domain reveals constraints on folding and ACE2 binding. Cell 182: 1295-1310.e20. 
79. Gu H et al., 2020. Adaptation of SARS-CoV-2 in BALB/c mice for testing vaccine efficacy. Science 369: 1603-1607.

80. Yurkovetskiy $L$ et al., 2020. Structural and functional analysis of the D614G SARS-CoV-2 spike protein variant. Cell 183: 739-751 e8.

81. Ku Z et al., 2021. Molecular determinants and mechanism for antibody cocktail preventing SARS-CoV-2 escape. Nat Commun 12: 1-13.

82. Wang $Q$ et al., 2020. Structural and functional basis of SARSCoV-2 entry by using human ACE2. Cell 181: 894-904.

83. Zhao $S$ et al., 2021. Inferring the association between the risk of COVID-19 case fatality and N501Y substitution in SARS-CoV2. Viruses 13: 638.

84. Waters E, Doyle J, 2004. Systematic reviews of public health in developing countries are in train. BMJ 328: 585.

85. Zhao $S$ et al., 2020. COVID-19 and gender-specific difference: analysis of public surveillance data in Hong Kong and Shenzhen, China, from January 10 to February 15, 2020. Infect Control Hosp Epidemiol 41: 750-751.

86. Torneri A, Libin P, Scalia Tomba G, Faes C, Wood JG, Hens N, 2021. On realized serial and generation intervals given control measures: the COVID-19 pandemic case. PLOS Comput Biol 17: e1008892.

87. Sun $\mathrm{K}$ et al., 2021. Transmission heterogeneities, kinetics, and controllability of SARS-CoV-2. Science 371: eabe2424.

88. Leung K, Wu JT, Liu D, Leung GM, 2020. First-wave COVID-19 transmissibility and severity in China outside Hubei after control measures, and second-wave scenario planning: a modelling impact assessment. Lancet 395: 1382-1393.
89. Ong SWX, Young BE, Lye DC, 2021. Lack of detail in population-level data impedes analysis of SARS-CoV-2 variants of concern and clinical outcomes. Lancet Infect Dis 21: 1195-1197.

90. Zhao S, Stone L, Gao D, Musa SS, Chong MKC, He D, Wang $\mathrm{MH}, 2020$. Imitation dynamics in the mitigation of the novel coronavirus disease (COVID-19) outbreak in Wuhan, China from 2019 to 2020. Ann Trans/ Med 8: 448.

91. Sun K, Chen J, Viboud C, 2020. Early epidemiological analysis of the coronavirus disease 2019 outbreak based on crowdsourced data: a population-level observational study. Lancet Digit Health 2: e201-e208.

92. Jentsch PC, Anand M, Bauch CT, 2021. Prioritising COVID-19 vaccination in changing social and epidemiological landscapes: a mathematical modelling study. Lancet Infect Dis (In press).

93. Liu Y, Morgenstern C, Kelly J, Lowe R, Jit M, 2021. The impact of non-pharmaceutical interventions on SARS-CoV-2 transmission across 130 countries and territories. BMC Med 19: $1-12$.

94. Li Y, Campbell H, Kulkarni D, Harpur A, Nundy M, Wang X, Nair H, for Covid UN, 2021. The temporal association of introducing and lifting non-pharmaceutical interventions with the time-varying reproduction number (R) of SARS-CoV-2: a modelling study across 131 countries. Lancet Infect Dis 21: 193-202.

95. Kraemer MUG et al., 2021. Spatiotemporal invasion dynamics of SARS-CoV-2 lineage B.1.1.7 emergence. Science 373: 889-895. 